\title{
Susac syndrome complicating a SARS-CoV-2 infection
}

\author{
Vincent Raymaekers ${ }^{1}$ (D) Simon D'hulst ${ }^{2} \cdot$ Dorien Herijgers $^{3} \cdot$ Johanna Vercammen $^{3}$ - Annelies Fabry ${ }^{4} \cdot$ Julie Dutoit $^{5}$. \\ Emmanuel D'Heygere $^{6} \cdot$ Evelien Vancaester $^{7} \cdot$ Patrick Vanderdonckt $^{7}$
}

Received: 25 January 2021 / Revised: 29 September 2021 / Accepted: 19 October 2021 / Published online: 4 November 2021

(c) Journal of NeuroVirology, Inc. 2021

\begin{abstract}
In 2020 the world was captivated by the COVID-19 pandemic. Current scientific evidence suggests an interaction of SARSCoV-2 and the human immune system. Multiple cases were reported of patients with COVID-19 presenting with encephalopathy, confusion or agitation, stroke, and other neurologic symptoms. We present a case of a 40-year-old man diagnosed with Susac syndrome after COVID-19, presenting with acute sensorineural hearing loss, encephalopathy, a splenial "snowball-like" lesion, and branch retinal artery occlusions with distal arterial wall hyperintensity. Although the pathophysiology of Susac syndrome remains unclear, this case is in line with the ongoing debate about the influence of SARS-CoV-2 on the human immune system. Corticosteroid treatment was initiated, followed by two treatments with rituximab, with clinical improvement of the symptomatology. Maintenance treatment currently consists of mycophenolic acid (MPA). Future research will need to focus on the underlying mechanisms for COVID-19-associated (autoimmune) complications.
\end{abstract}

Keywords COVID-19 $\cdot$ Susac syndrome $\cdot$ Snowball-like lesion

\section{Background}

The WHO declared COVID-19 to be a pandemic as of March 2020 (WHO Director-General 2020). As of writing this report, there is still a high rate of new SARS-CoV-2 infections worldwide (World Health Organization 2020).

Recent scientific evidence reports a wide range of central nervous system (CNS) complications associated with COVID-19. Multiple cases were reported of patients with COVID-19 presenting with encephalopathy, confusion or agitation, stroke, and other neurologic symptoms (Ellul et al. 2020; Helms et al. 2020).

Susac syndrome is a rare condition, first described by the American neurologist John Susac in 1979, characterized by a

Vincent Raymaekers

Vincent.raymaekers@gmail.com

1 University of Antwerp, Antwerp, Belgium

2 University of Ghent, Ghent, Belgium

3 University of Leuven, Leuven, Belgium

4 Department of Ophthalmology, AZ Groeninge, Kortrijk, Belgium

5 Department of Radiology, AZ Groeninge, Kortrijk, Belgium

6 Department of Otolaryngology, Head and Neck Surgery, AZ Groeninge, Kortrijk, Belgium

7 Department of Neurology, AZ Groeninge, Kortrijk, Belgium typical triad of encephalopathy, sensorineural hearing loss, and branch retinal artery occlusions (BRAO) (Dörr et al. 2013). To date, there is only one case report on Susac syndrome following a SARS-CoV-2 infection (Venditti et al. 2020).

Here, we present a case of a 40-year-old man diagnosed with Susac syndrome after COVID-19, presenting with the classic triad. Although the pathophysiology of Susac syndrome remains unclear, this case is in line with the ongoing debate about the influence of SARS-CoV-2 on the human immune system.

\section{Case presentation}

A 40-year-old male patient, with no relevant medical history, was referred to the emergency department with acute hearing loss, tinnitus, an abnormal gait pattern, and progressive confusion for the past 7 days. He had tested positive for SARS-CoV-2 14 days prior to presentation at the hospital.

History taking was seriously complicated by the acute confusion and severe hearing problems. The patient was unaware of the situation and unable to tell his name, age, or location. Heteroanamnesis only revealed complaints of vertigo and a mild headache in the past days. There was an uncoordinated atactic gait pattern and lower facial asymmetry. Confrontational visual field testing was negative for visual field defects. There were no other obvious objective signs of neurological deficits. 

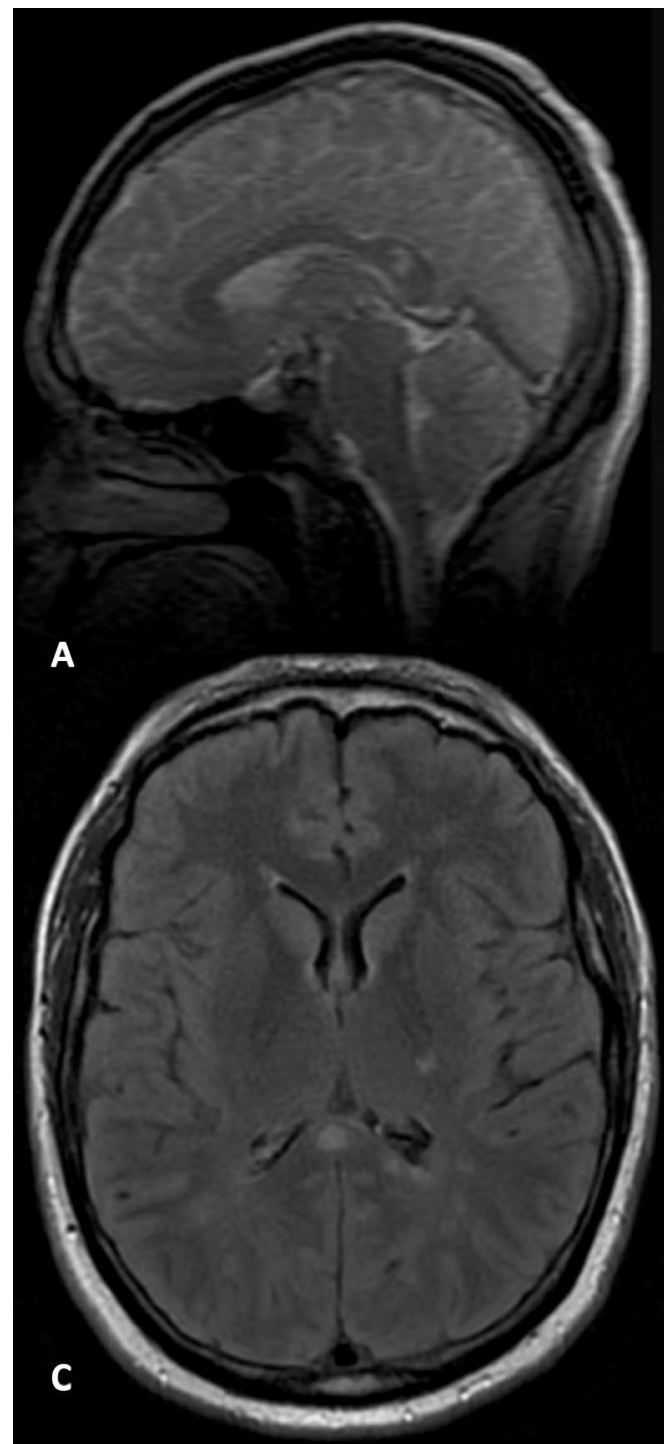

B
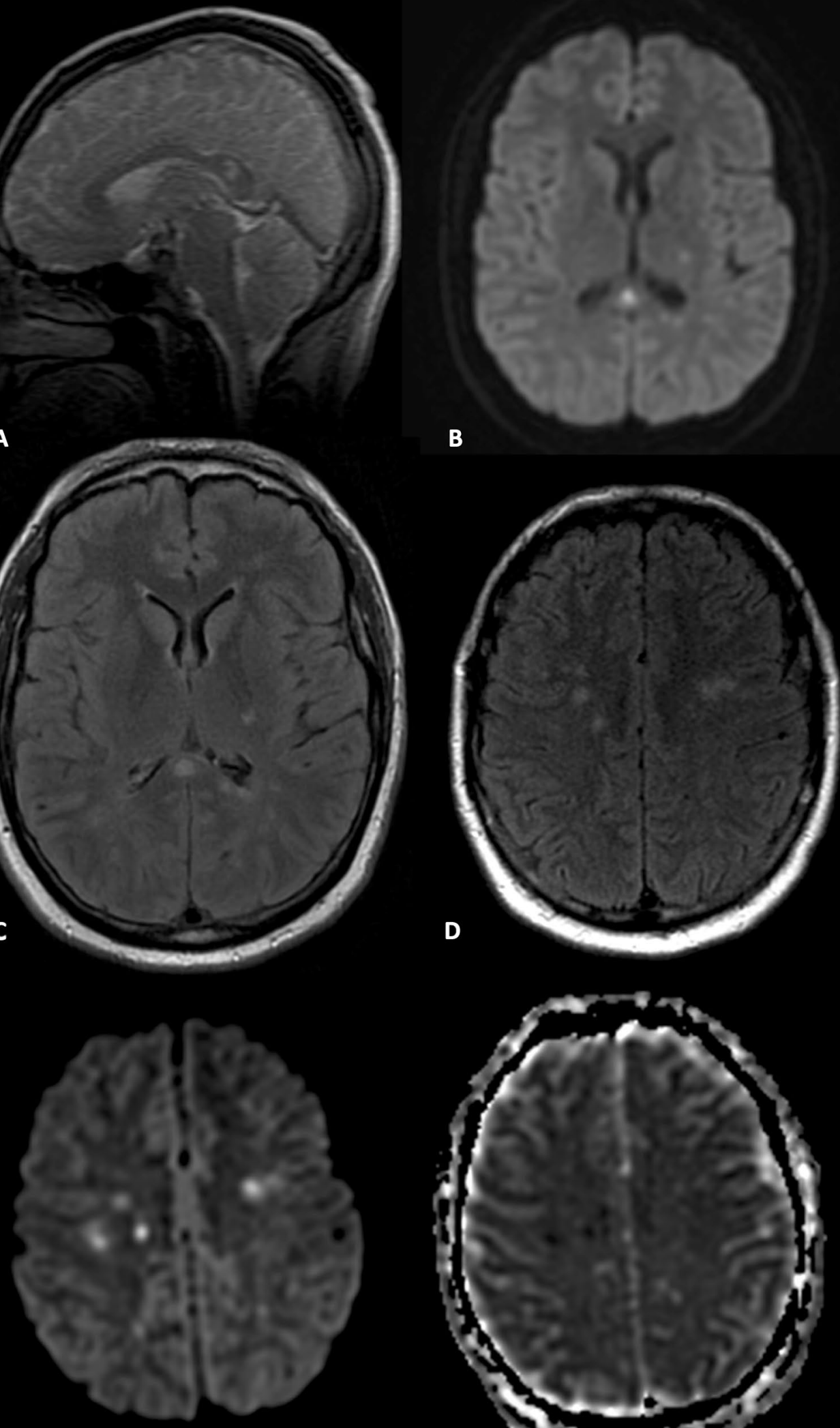

E

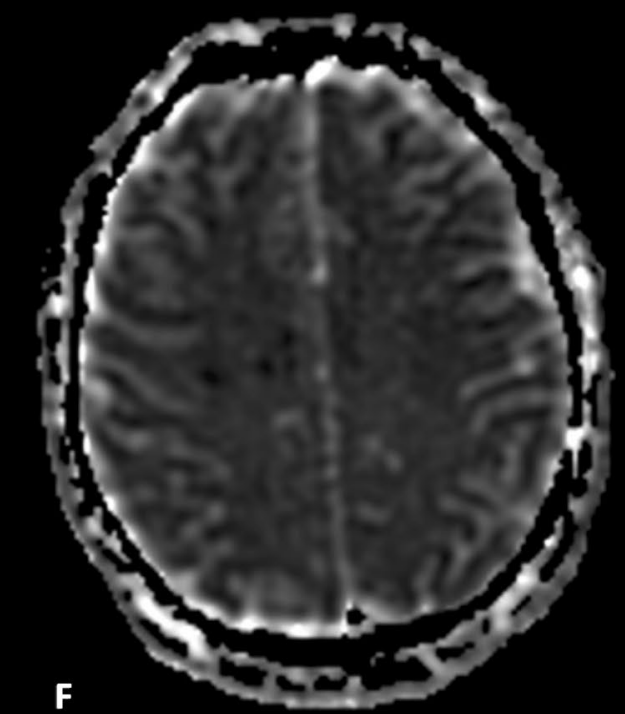


4Fig. 1 Magnetic resonance imaging (MRI) after 7 days (diagnosis) with the characterizing splenial lesion of the corpus callosum on sagittal T2 localizer (A), DWI b1000 image (B), and T2 axial FLAIR (C), together with bilateral supratentorial enhancing white matter lesions, already present on the first MRI, on FLAIR (D), DWI b1000 $(\mathbf{E})$, and ADC imaging $(\mathbf{F})$

\section{Investigations}

Upon admission, the patient still had a positive nasopharyngeal polymerase chain reaction (PCR) assay for SARSCoV-2. However, the viral load was low and considered residual viral particles. The complete blood count showed a normal red and white blood cell count with normal distribution and thrombocytosis.

The initial cerebral MRI was complicated by moving artifacts due to confusion. It showed hyperintense lesions on DWI and FLAIR anteriorly in the splenium of the corpus callosum and posterior commissure with bilateral supratentorial diffusion-restricted white matter lesions (Fig. 1). Radiologically there were arguments for a, possibly COVID-19 induced, mild encephalopathy with reversible splenial lesion (MERS).

Cerebrospinal fluid analysis showed an elevated total protein $(1990 \mathrm{mg} / \mathrm{L})$, albumin $(1450 \mathrm{mg} / \mathrm{L}), \operatorname{IgG}(235 \mathrm{mg} / \mathrm{L})$, and minimal pleocytosis $(6 \mathrm{WBC} / \mu \mathrm{L})$. Bacterial cultures, SARS-CoV-2 PCR, and multiplex PCR on CSF were negative.

A second cerebral MRI scan was performed 7 days later. The supratentorial diffusion-restricted T2 FLAIR hyperintense lesions were comparable .The lesion in the splenium of the corpus callosum had now decreased in volume and looked more like a "snowball" in the sagittal plane (Fig. 1).

Next, audiometric tests were performed to objectify the hearing loss. Audiometry showed a severe hearing impairment diagnosed as sensorineural hearing loss. Thresholds based on audiometry were found to be $45 \mathrm{~dB}$ at $500 \mathrm{~Hz}$ and $70 \mathrm{~dB}$ at $1-4 \mathrm{kHz}$ in the right ear and $45 \mathrm{~dB}$ at $500 \mathrm{~Hz}, 70 \mathrm{~dB}$ at $1 \mathrm{kHz}$, and $60 \mathrm{~dB}$ at $2-4 \mathrm{kHz}$ in the left ear. However, brainstem evoked response audiometry (BERA) revealed normal bilateral thresholds of 20dB. Otoacoustic emissions (OAE) were absent bilaterally.

Ophthalmological examination demonstrated normal visual acuity, normal eye pressure, and a normal anterior segment. However, fluorescein angiography within the first week and after 3 weeks visualized BRAOs in several areas of the left and right eye, with arteriolar wall hyperfluorescence (AWH) (Fig. 2). No improvement of the arterial occlusions was visualized after 3 weeks. Optical coherence tomography (OCT) demonstrated a normal thickness of the retinal nerve fiber layer (RNFL) to the outer plexiform layer.

The combination of acute sensorineural hearing loss, encephalopathy, the presence of a splenial "snowball-like" lesion, and BRAOs with distal AWH confirmed the diagnosis of Susac syndrome.

\section{Treatment}

Initially, treatment with acyclovir and remdesivir was initiated for the, potentially SARS-CoV-2 associated, encephalitis (Beigel et al. 2020). When Susac syndrome was confirmed, therapy was adjusted. Intravenous methylprednisolone $(1000 \mathrm{mg}$ ) was administered for 5 days, followed by an oral dose of $64 \mathrm{mg}$ daily tapered over 5 weeks. A $16 \mathrm{mg}$ maintenance treatment was introduced in association with aspirin. In addition, after the 5 days of methylprednisolone therapy, a one-day treatment with a monoclonal antibody, Rituximab $1000 \mathrm{mg}$ was administered. The administration of rituximab $1000 \mathrm{mg}$ was repeated after 2 weeks. In cooperation with a tertiary university hospital, maintenance treatment with mycophenolic acid was associated 2 months after the initial diagnosis to reduce corticosteroid use.

\section{Outcome and follow-up}

The patient remained hospitalized for 15 days. After initiating the methylprednisolone therapy, a significant improvement of gait and encephalopathic symptoms was objectified. One month after discharge, the patient was evaluated. He was less confused and had problems with concentrating. There was an objective improvement in hearing based on the audiometry after 1 month (Fig. 3) when comparing the hearing thresholds mentioned before.

Check-up at 2 months showed residual physical deconditioning, intermittent vertigo, and persistent tinnitus on the right side. In general, the patient was doing much better. Audiometry was worse on the right side at low frequencies and remained stable on the left side. A new angiography after 3 months showed residual BRAOs with even a deterioration of arterial occlusions (Fig. 2).

\section{Discussion}

We presented a case of Susac syndrome complicating a recent SARS-CoV-2 infection. Today, only one case has been reported by Venditti et al. on Susac syndrome following a SARS-CoV-2 infection (Venditti et al. 2020). Unlike in our current case, the classic triad was not present. Neurological signs similarly improved significantly after administration of IV methylprednisolone. Here, the initial differential diagnosis consisted of herpes encephalitis and COVID19-induced MERS. The final diagnosis of Susac syndrome was facilitated by a multidisciplinary diagnostic work-up by 

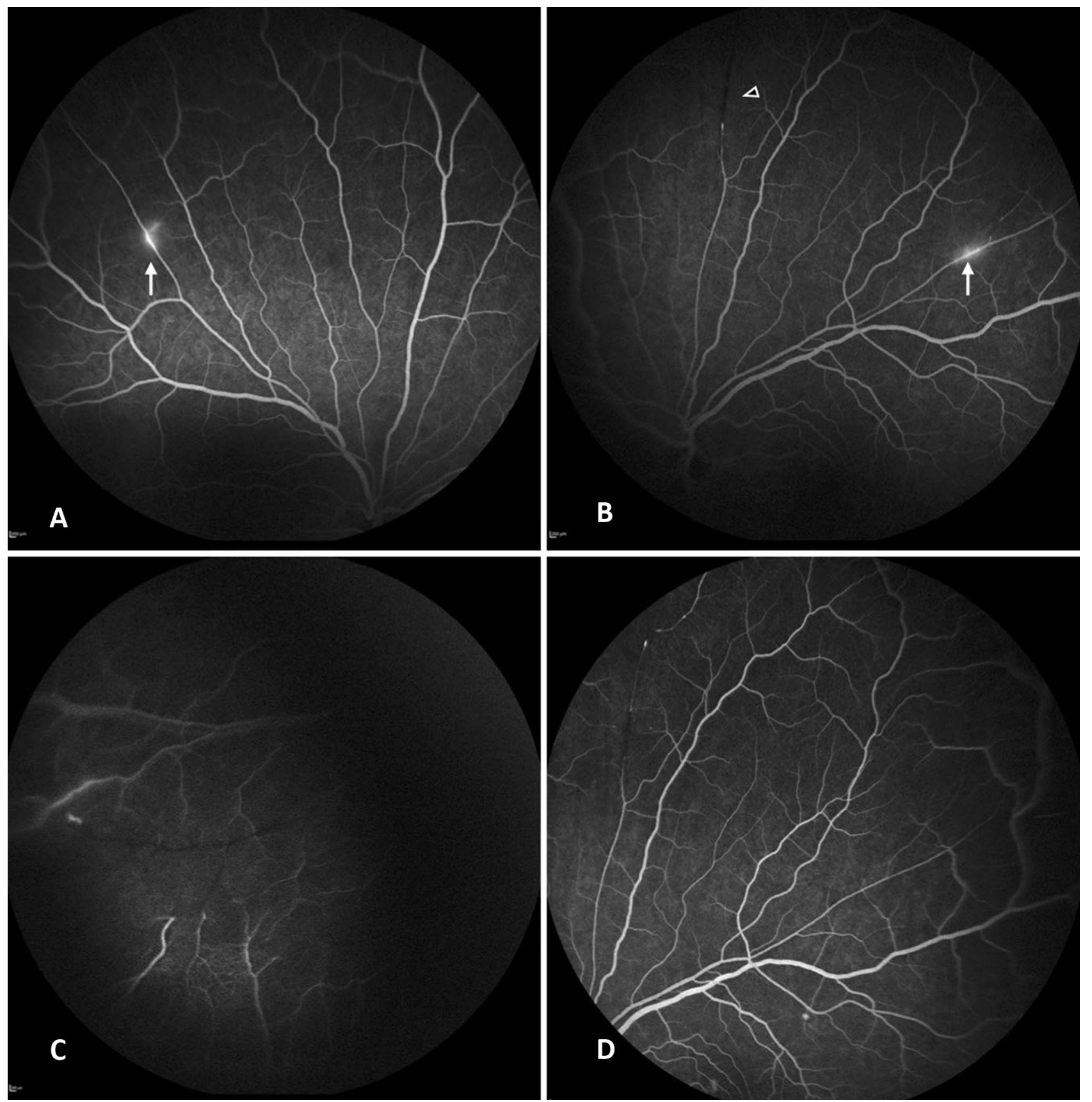

Fig. 2 Fluorescein angiography within the first week and after 3 weeks visualized comparable BRAOs (arrowhead) in the superior and nasal area of the right eye, with AWH in the superior and inferior area. The left eye showed BRAOs in the superior, temporal and

the neurologist, neuroradiologist, ophthalmologist, and ENT specialist (Egan 2019; Kleffner et al. 2016). When submitting this case report, the total follow-up time was only 14 weeks. Further frequent evaluation by the multidisciplinary team was planned.

Numerous cases of neurological complications associated with the current COVID-19 pandemic have been reported

nasal area, with a superior zone of AWH in the first fluorescein angiography (white arrows) (A and $\mathbf{B}$ ). A new angiography after 3 months showed residual BRAOs with even a deterioration of local arterial occlusions (C and D)

(Ellul et al. 2020). The pathophysiology of Susac syndrome remains unclear but may be mediated by an autoimmune response, causing microvessel ischemic occlusions in cerebral, cochlear, and retinal tissue. It is questionable if the recent SARS-CoV-2 infection is causal or coincidental. However, SARS-CoV-2 interferes with the angiotensinconverting enzyme 2 (ACE-2) receptor, contributing 
RIGHT EAR

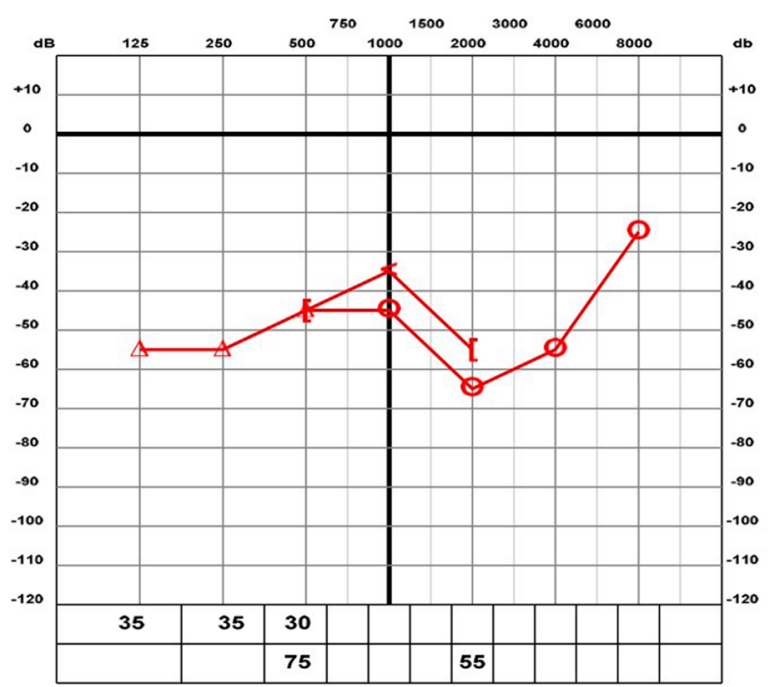

Fletcher index $R=47 \mathrm{~dB}-\mathrm{L}=28 \mathrm{~dB}$ IPA indox $R=46 \mathrm{~dB}, L=31 \mathrm{~dB}$ BIAP index $R=49 \mathrm{~dB} \cdot L=38 \mathrm{~dB}$
LEFT EAR

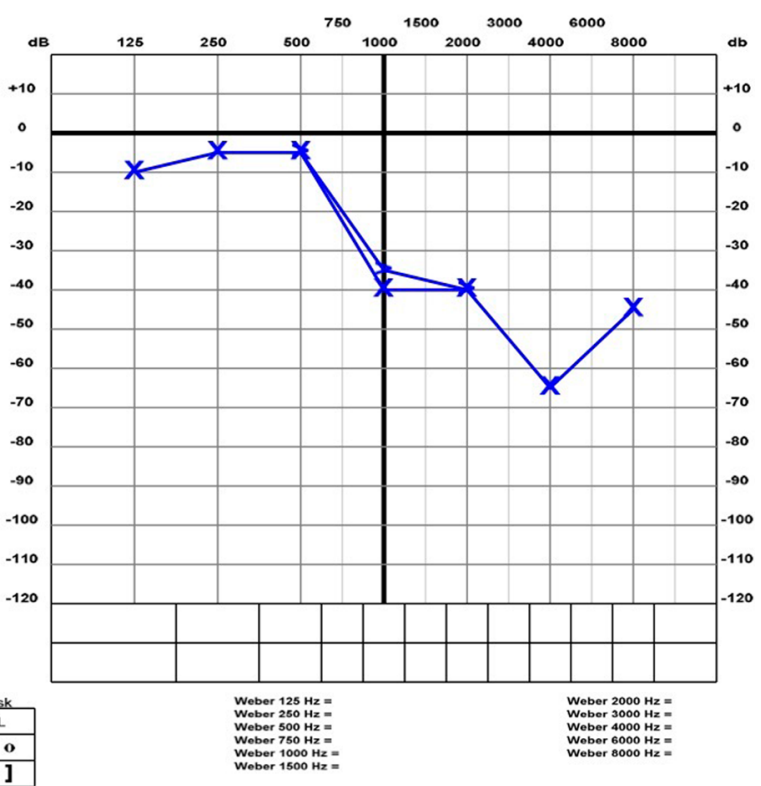

Fig. 3 Audiometry 1 month after the initial symptoms. Thresholds were $40 \mathrm{~dB}$ at $500 \mathrm{~Hz}, 50-60 \mathrm{db}$ at $1-4 \mathrm{kHz}$, and $25 \mathrm{~dB}$ at $8 \mathrm{kHz}$ in the right ear and $5 \mathrm{~dB}$ at $250 \mathrm{~Hz}, 5 \mathrm{~dB}$ at $500 \mathrm{~Hz}, 40 \mathrm{~dB}$ at $1-2 \mathrm{kHz}, 60 \mathrm{~dB}$ at $4 \mathrm{kHz}$, and $50 \mathrm{~dB}$ at $8 \mathrm{kHz}$ in the left ear. The initial audiometry was not interpretable.

to endothelial dysfunction and microvessel occlusion. Postmortem high-resolution MRI of the brains of patients with COVID-19 confirmed small vessel damage (Lee et al. 2020). COVID-19-associated strokes are ascribed to a severe introduced coagulopathy and large vessel occlusion combined with systemic inflammation and cytokine storm (Fifi and Mocco 2020). This cytokine storm could potentially trigger the human immune system and induce autoimmunity. In a retrospective study by Wilf-Yarkoni et al., infectious serological findings for cytomegalovirus suggest a post infectious mechanism, as might be the case after a COVID-19 infection (Wilf-Yarkoni et al. 2020). Furthermore, viral infections have previously been mentioned in other autoimmune-mediated diseases such as Epstein-Barr virus in multiple sclerosis (Icenogle 2020; Ehrenfeld et al. 2020). As this is currently an important topic in scientific research, future research will need to focus on the underlying mechanisms for COVID-19-associated (autoimmune) complications.

\section{Learning points}

- COVID-19 can be associated with neurological complications such as stroke and encephalopathy.

- Despite insufficient evidence, Susac syndrome may be triggered by an autoimmune response associated with a SARS-CoV-2 infection.
- The diagnosis of Susac syndrome requires a multidisciplinary work-up.

- Future research should focus on exposing the underlying mechanism of the interference of SARS-CoV-2 with the human immune system.

\section{Declarations}

Conflict of interest The authors declare no competing interests.

\section{References}

Beigel JH, Tomashek KM, Dodd LE, Mehta AK, Zingman BS, Kalil AC et al (2020) Remdesivir for the treatment of Covid-19 — final report. N Engl J Med 383(19):1813-1826

Dörr J, Krautwald S, Wildemann B, Jarius S, Ringelstein M, Duning $\mathrm{T}$ et al (2013) Characteristics of Susac syndrome: a review of all reported cases. Nat Rev Neurol 9(6):307-316

Egan RA (2019) Diagnostic criteria and treatment algorithm for Susac syndrome. J Neuroophthalmol 39(1):60-67

Ehrenfeld M, Tincani A, Andreoli L, Cattalini M, Greenbaum A, Kanduc D et al (2020) Covid-19 and autoimmunity. Autoimmun Rev 19(8):102597

Ellul MA, Benjamin L, Singh B, Lant S, Michael BD, Easton A et al (2020) Neurological associations of COVID-19. Lancet Neurol 19(9):767-783

Fifi JT, Mocco J (2020) COVID-19 related stroke in young individuals. Lancet Neurol 19(9):713-715 
Helms J, Kremer S, Merdji H, Clere-Jehl R, Schenck M, Kummerlen C et al (2020) Neurologic features in severe SARS-CoV-2 infection. N Engl J Med 382(23):2268-2270

Icenogle T (2020) COVID-19: infection or autoimmunity. Front Immunol 11:2055

Kleffner I, Dörr J, Ringelstein M, Gross CC, Böckenfeld Y, Schwindt W et al (2016) Diagnostic criteria for Susac syndrome. J Neurol Neurosurg Psychiatry 87(12):1287

Lee M-H, Perl DP, Nair G, Li W, Maric D, Murray H et al (2020) Microvascular injury in the brains of patients with Covid19. N Engl J Med

Venditti L, Rousseau A, Ancelet C, Papo T, Denier C (2020) Susac syndrome following COVID-19 infection. Acta Neurol Belg 1-3

WHO Director-General (2020) WHO Director-General's opening remarks at the media briefing on COVID-19. Available from: https://www.who.int/director-general/speeches/detail/ who-director-general-s-opening-remarks-at-the-mediabriefing-on-covid-19---11-march-2020

Wilf-Yarkoni A, Elkayam O, Aizenstein O, Oron Y, Furer V, Zur D et al (2020) Increased incidence of Susac syndrome: a case series study. BMC Neurol 20(1):332

World Health Organization (2020) Global research on coronavirus disease (COVID-19). Available from: https://www.who. $\mathrm{int/emergencies/diseases/novel-coronavirus-2019/global-}$ research-on-novel-coronavirus-2019-ncov

Publisher's Note Springer Nature remains neutral with regard to jurisdictional claims in published maps and institutional affiliations. 